\title{
Shape Matching and Recognition Using Group-Wised Points
}

\author{
Junwei Wang, Yu Zhou, Xiang Bai, and Wenyu Liu* \\ Department of Electronics and Information Engineering, \\ Huazhong University of Science and Technology, Wuhan, China \\ wjw. 20012001@163. com, zhouyu. hust@gmail.com, xiang.bai@gmail.com, \\ liuwy@hust.edu.cn
}

\begin{abstract}
Shape matching/recognition is a very critical problem in the field of computer vision, and a lot of descriptors and methods have been studied in the literature. However, based on predefined descriptors, most of current matching stages are accomplished by finding the optimal correspondence between every two contour points, i.e., in a pair-wised manner. In this paper, we provide a novel matching method which is to find the correspondence between groups of contour points. The points in the same group are adjacent to each other, resulting in a strong relationship among them. Two groups are considered to be matched when the two point sequences formed by the two groups lead to a perfect one-toone mapping. The proposed group-wised matching method is able to obtain a more robust matching result, since the co-occurrence (order) information of the grouped points is used in the matching stage. We test our method on three famous benchmarks: MPEG-7 data set, Kimia's data set and Tari1000 data set. The retrieval results show that the new group-wised matching method is able to get encouraging improvements compared to some traditional pair-wised matching approaches.
\end{abstract}

Keywords: Shape matching, Pair-wised matching, Group-wised, Co-occurrence.

\section{Introduction}

Shape description and matching is a very critical problem in the field of computer vision. There are some important issues to be noted. First, geometric transformation invariance should be satisfied when matching two shapes, i.e., one shape and its translated/rotated/scaled versions are supposed to be very similar. Next, some intra-class variations, such as noise, articulation, local deformation and occlusion, should be carefully treated. The influences of these variances to shape similarity measure should be decreased to an acceptable level.

To handle the complex situations listed above, several shape representations and descriptors with "rich" shape information have been studied in the last decade, including Visual Part [2], Shape Context (SC) [3], Inner-distance Shape

\footnotetext{
^ Corresponding author.
} 
Context (IDSC) [9], Triangle Area Representation (TAR) [1], and Shape Tree [10]. Latecki and Lakamper introduced one novel shape representation called Visual Part 2. Shapes are simplified by a process of digital curve evolution (DCE) and are further decomposed into perceptually meaningful parts, which are called Visual Parts. Shape matching is performed by looking for the optimal correspondence of visual parts. Shape Context [3], which utilizes the geometric relationship between contour sample points, is one of the most classical shape descriptors in the literature. For each sample point on one shape contour, its Shape Context captures the spatial distribution of all the other sample points relative to it, which offers a globally discriminative characterization. Ling and Jacobs proposed a novel distance definition called inner distance as a replacement for the usual Euclidean distance. The inner distance is defined as the length of the shortest path between landmark points within the shape silhouette, which is articulation insensitive and more effective at capturing part structures. Using the inner distance, Shape Context can be extended to a novel descriptor called Inner-Distance Shape Context 9]. Triangle Area Representation [1] presents a measure of convexity/concavity of each contour point using the signed areas of triangles formed by boundary points at different scales. The area value of triangle is a measure for the curvature of corresponding contour point. This representation is effective in capturing both local and global shape characteristics. Shape Tree [10] is one classical segment-based shape matching algorithm, which proposed a hierarchical representation for contour curves. The original curve can be broken into two halves by the middle point on it, and each of the two sub-curves can be broken into its halves. This hierarchical description can be represented by a binary tree, which is called the Shape Tree of a curve. The matching process is performed by comparing the hierarchical segments explicitly.

The methods introduced in the above paragraph are all effective shape descriptors. However, among all these methods, the matching stage is accomplished by finding the optimal correspondence between contour sample points or local parts. The sample points or shape parts, which are represented by the predefined descriptors, mainly consist of local characteristics of given shapes. For example, Shape Context, which is one of the most classical descriptors in recent years, pays more attention to local shape features. The bins in the histogram of Shape Context are uniform in a log-polar space, which makes it much more sensitive to nearby sample points than to points farther away [3]. Consequently, the contour sample points or parts are matched together probably because the two points or parts have similar local shape characteristics. This kind of matching strategy is sometimes not robust enough to deal with complex situations, when there are certain amount of inter/intra class variations.

In this paper, we present a novel method to achieve more robust shape matching. Our motivation comes from this observation: two contour points should take a correspondence, not only because these two points are very similar, but also the points related to them are very similar as well. If we take related points into consideration, the matching result is much more likely to be correct. Therefore, we put related points into one group, and when we perform matching between 
two points, we consider matching their corresponding groups. We call this novel and robust matching strategy group-wised matching. In the next section, we will give some cues on how to judge which are related points and how to define groups.

A similar manner to our idea is the Dynamic Programming (DP) algorithm, which has been widely used for shape matching (e.g., by the method of Multiscale Convexity Concavity (MCC) [6] and Inner-Distance Shape Context (IDSC) [9, etc.). In DP algorithm, the order information of contour sample points is utilized as a global constraint for optimal correspondence. However, in current DP algorithm, local point-wise misalignment is allowed, and the correspondence is still found between single points, not group of points. We combine our groupwised matching approach with the usual DP algorithm, and achieved better retrieval results (see experiments in Section 3 ).

The remainder of this paper is organized as follows. Section 2 introduces the proposed group-wised shape matching algorithm in details. Section 3 presents the experimental results. Finally, Section 4 makes some conclusions.

\section{The Proposed Group-Wised Method}

In this Section, we give the definition what is a group and the group-wised matching method in details.

\subsection{Point Groups}

Let $\mathbf{S}=\left\{\mathbf{p}_{i}\right\}(i=1, \ldots, N)$ denotes the sequence of equidistant sample points on the outer contour of a given shape $\mathbf{S}$, where the index $i$ is according to the order of the sample points along the contour in the counter-clockwise direction. In our implementation, we set the number $N=100$, which is consistent with the settings in many recent works, such as [6] and 9 .

To build up the group-wised matching strategy, at first we need to give the definition of group. As stated in the previous section, it is a good choice to combine one given point and its related points on the shape contour to form a group. Then the problem is which point is related to the given point. We adopt one simple strategy here: the neighboring points are treated as the related points. That is because related points are more likely to get close to each other than get far away from each other. Just take the shape boundary of a human-being as an example. The contour points representing his/her "ears", "eyes", "nose" and "mouth" are closely related to each other, as all of them are the elements of the "head". On the contrary, the contour points representing his/her "hands" and "feet" are almost unrelated to each other. Figure 1 gives another example of related points on the boundary of a horse shape. In Figure 11, some related points on the head of the horse are labeled as red dots connected by solid lines; some unrelated points on its legs and back are labeled as blue dots connected by dashed lines.

For the reason stated above, we choose points close to each other to form a group. Specifically, for one sample point $\mathbf{p}_{i}$, we choose $k$ nearby points on 


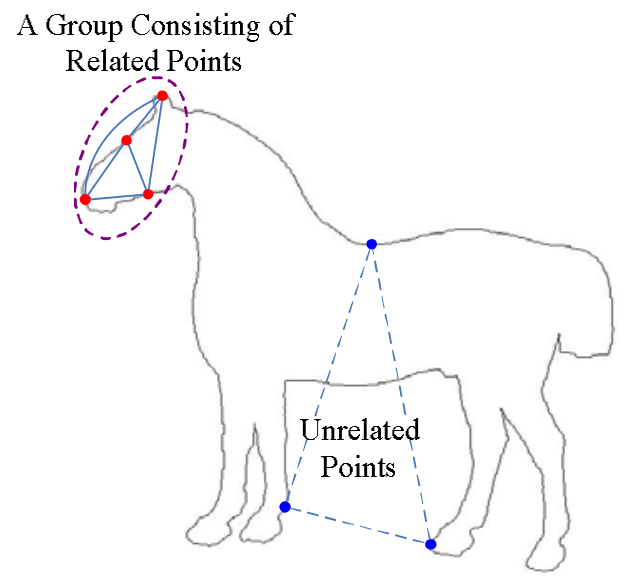

Fig. 1. An example of related/unrelated points. The "horse" shape is chosen from MPEG-7 CE-Shape-1 part B database 1]. See more details in the text.

the left hand side $\mathbf{p}_{i-1}, \mathbf{p}_{i-2}, \ldots, \mathbf{p}_{i-k}$, and $k$ nearby points on the right hand side $\mathbf{p}_{i+1}, \mathbf{p}_{i+2}, \ldots, \mathbf{p}_{i+k}$. Note that the number of points on the left hand side is equivalent to the number of points on the right hand side, which leads to a balance description. With these $2 k+1$ points, we are able to define a meaningful point group, $G_{i}$. The point $\mathbf{p}_{i}$ is right on the center of this group, which is regarded as the centroid of the group $G_{i}$. As there is co-occurrence (order) information among these contour sample points, the point group $G_{i}$ is indeed a point subsequence belonging to the point sequence $\left\{\mathbf{p}_{i}\right\}(i=1, \ldots, N)$ on the outer contour of the shape $\mathbf{S}$ :

$$
G_{i}=\left(\mathbf{p}_{i-k}, \mathbf{p}_{i-k+1}, \ldots, \mathbf{p}_{i-1}, \mathbf{p}_{i}, \mathbf{p}_{i+1}, \ldots, \mathbf{p}_{i+k-1}, \mathbf{p}_{i+k}\right) .
$$

The parameter $k$, which controls the scale or the point number of the group, is regarded as the radius of the group. When $k$ increases, the neighborhood of the point $\mathbf{p}_{i}$ increases, and more points (with more shape characteristics) are included into the group.

For every sample point $\mathbf{p}_{i}(i=1, \ldots, N)$, we define a point group $G_{i}(i=$ $1, \ldots, N)$. Then the whole shape $\mathbf{S}$ is represented by a sequence of point groups:

$$
\mathcal{G}=\mathcal{G}(\mathbf{S})=\left(G_{1}, G_{2}, \ldots, G_{N}\right)
$$

\subsection{Group-Wised Matching}

For the task of shape recognition, usually a shape similarity or dissimilarity (distance) is computed by finding the optimal correspondence of contour points. Then the shape dissimilarity is the sum of the distances of the corresponding points. As defined in Section 2.1, every sample point is represented by one group consisting of itself and several neighboring points. Consequently, the shape 
similarity is computed by finding the optimal correspondence of the predefined groups, and the shape dissimilarity value is the sum of the distances of the corresponding groups.

To match two shapes $\mathbf{X}, \mathbf{Y}$ (represented by two sequences of point groups), the dissimilarity between any pair of points (indeed groups) should be computed. Let $\mathbf{p}_{i}, \mathbf{q}_{j}$ denote contour points of $\mathbf{X}, \mathbf{Y}$, respectively, and $G_{i}, G_{j}$ denote corresponding groups for the two points. When we perform a matching between these two sample points $\mathbf{p}_{i}, \mathbf{q}_{j}$, we indeed perform a matching between the two groups $G_{i}, G_{j}$. To match the two groups, we consider not only the cost for mapping the two center points (i.e., the centroid of the groups) $\mathbf{p}_{i}, \mathbf{q}_{j}$, but also the costs for mapping their neighboring points within corresponding groups. Specifically, if the original pair-wised cost (feature distance) for mapping two points $\mathbf{p}_{i}, \mathbf{q}_{j}$ $(i, j=1, \ldots, N)$ is denoted as $c\left(\mathbf{p}_{i}, \mathbf{q}_{j}\right)$, then our group-wised matching method defines the mapping cost for the two groups $G_{i}, G_{j}$ as:

$$
d\left(\mathbf{p}_{i}, \mathbf{q}_{j}\right)=d\left(G_{i}, G_{j}\right)=c\left(\mathbf{p}_{i}, \mathbf{q}_{j}\right)+\sum_{t=1}^{k} \omega_{t}\left\{c\left(\mathbf{p}_{i-t}, \mathbf{q}_{j-t}\right)+c\left(\mathbf{p}_{i+t}, \mathbf{q}_{j+t}\right)\right\}
$$

In this way, shape matching is achieved through a group-wised manner. That is, not only the feature distance between the two points $\mathbf{p}_{i}, \mathbf{q}_{j}$ but also the feature distances between the neighboring points of the two points $\mathbf{p}_{i}, \mathbf{q}_{j}$ are utilized. Clearly, the pair-wised cost information $c\left(\mathbf{p}_{i}, \mathbf{q}_{j}\right)$ is completely included in the group-wised cost $d\left(\mathbf{p}_{i}, \mathbf{q}_{j}\right)$.

Note that the co-occurrence (order) information of every point group is perfectly included into the mapping cost defined in Equation 3. as we assume that the two groups $G_{i}$ and $G_{j}$ must have a perfect one-to-one mapping in their sequence point order, i.e., $\mathbf{p}_{i-t}$ corresponds to $\mathbf{q}_{j-t}$ and $\mathbf{p}_{i+t}$ corresponds to $\mathbf{q}_{j+t}$ for all $t=1, \ldots, k$. Figure 2 shows the idea of our group-wised matching method along with the difference between group-wised and pair-wised approaches.

This group-wised mapping cost can also be regarded as one novel pair-wised mapping cost for each pair of two points: the original pair-wised cost $c\left(\mathbf{p}_{i}, \mathbf{q}_{j}\right)$ is replaced by the novel pair-wised cost $d\left(\mathbf{p}_{i}, \mathbf{q}_{j}\right)$ for all $i, j=1, \ldots, N$.

In Equation 3, $\omega_{t}$ is the weight coefficient for every neighboring point. In order to be able to tolerate boundary deformations, the costs of mapping points closer to the two points $\mathbf{p}_{i}, \mathbf{q}_{j}$ are treated as more important than the costs of mapping points farther away from $\mathbf{p}_{i}, \mathbf{q}_{j}$. To achieve this, the value of $\omega_{t}$ should become smaller and smaller (approaching zero) when $t$ is increasing. In our implementations, the weights of neighboring points are set to decline exponentially, i.e., the weight coefficients are defined as follows:

$$
\omega_{t}=\frac{1}{2^{t}}
$$

According to Equation 3, we calculate the novel mapping cost for every pair of sample points $\mathbf{p}_{i}, \mathbf{q}_{j}(i, j=1, \ldots, N)$. With these costs, we obtain a cost matrix $\mathbf{D}(\mathbf{X}, \mathbf{Y})$ for the two shapes $\mathbf{X}, \mathbf{Y}$ : 


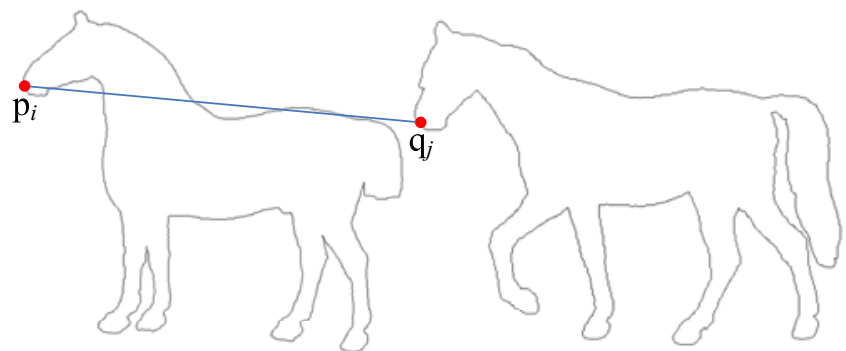

(a)

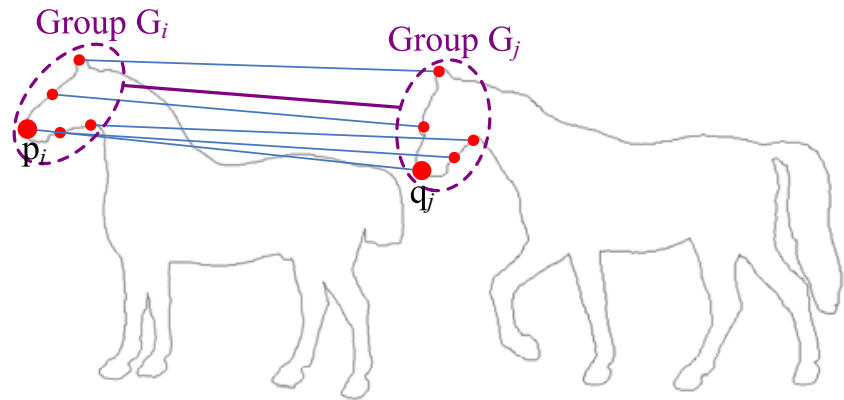

(b)

Fig. 2. An example of (a) pair-wised matching and (b) group-wised matching. Note that in our group-wised matching approach the points follow a perfect one-to-one mapping according to the order of the sample points along the shape contours.

$$
\mathbf{D}(\mathbf{X}, \mathbf{Y})=\left(d_{i, j}\right)=\left(d\left(\mathbf{p}_{i}, \mathbf{q}_{j}\right)\right), i, j=1, \ldots, N .
$$

$\mathbf{D}(\mathbf{X}, \mathbf{Y})$ is the input for any shape matching algorithm. In our method, we choose the Dynamic Programming (DP) algorithm as the matching method.

\subsection{Analysis}

Here we give a brief analysis for our method. As introduced in precious sections, the contribution of our method is a more robust matching approach, which results from including more local shape characteristics and the co-occurrence (order) information of contour points into the matching stage. As we see, the original shape descriptor is undefined, thus the original feature distance $\left(c\left(\mathbf{p}_{i}, \mathbf{q}_{j}\right)\right)$ is also undefined; this implies that there is no constraint to the original shape descriptor for sample points or local parts. This makes our method very easy to be combined with many current shape descriptors.

There is another valuable property for our method. According to Equation 3 , our method can also be regarded as a novel descriptor, which is simply the combination (sequence) of several predefined descriptors. That is, for every sample point $\mathbf{p}_{i}(i=1, \ldots, N)$, we introduce a novel descriptor $H_{i}$ : 


$$
H_{i}=\left(F_{i-k}, F_{i-k+1}, \ldots, F_{i-1}, F_{i}, F_{i+1}, \ldots, F_{i+k-1}, F_{i+k}\right),
$$

where $F_{t}(t=i-k, \ldots, i+k)$ is the predefined descriptor for the $t$ th sample point within the group $G_{i}$. Then the group-wised mapping cost defined in Equation 3 is just a novel pair-wised mapping cost as the feature distance between the two sample points $\mathbf{p}_{i}, \mathbf{q}_{j}$ based on their novel descriptors $H_{i}, H_{j}$ :

$$
d\left(\mathbf{p}_{i}, \mathbf{q}_{j}\right)=c\left(H_{i}, H_{j}\right)=\sum_{t=-k}^{k} c\left(F_{i+t}, F_{j+t}\right) .
$$

In this way, our method is able to inherit the properties of the primary descriptors. For example, if we adopt the primary descriptor as Shape Context, then the group-wised manner automatically obtains all the properties of Shape Context. This property makes our method very convenient when facing different applications as long as we are able to change the predefined descriptors freely.

The computational complexity of our method remains unchanged compared with the predefined descriptors. This results from Equation 3, which implies that in our method the only work we need to do is to sum up some original costs (feature distance) together to create some new costs. Since for every sample point, the number of costs being added together is the same (which is determined by the parameter $k$ ), this process can be implemented by matrix operations, whose computational complexity is even much lower than the complexity of feature distance computation. As a result, our group-wised algorithm doesn't bring any additional computational burden to the whole shape matching system, although it will make some more computational demands.

\section{Experiments}

This section gives the experimental results using our method. Without losing the generality of our method, the predefined descriptor is chosen as Shape Context (SC) [3], one of the most classical descriptors in the literature. The most important parameter in our method is the group radius $k$. In our implementations, $k$ is equal to 2 for all situations. We test the effect of the proposed method with three widely used shape databases, i.e., MPEG-7 data set [1, Tari1000 data set [13] and Kimia's 99 data set [5].

\subsection{MPEG-7 Shape Database}

The database of MPEG-7 CE-Shape-1 part B [1] is very famous in shape matching and classification. This database consists of 1,400 binary images from 70 shape categories, i.e., 20 images per category. This data set is rather difficult since there are some large intra-class variances. Some examples of this data set are given in Figure 3. Following the common performance measurement "bullseye test" 6] [9] [1] [3] we treat every image in this database as a query, and count the number of correct images in the top 40 matches. 


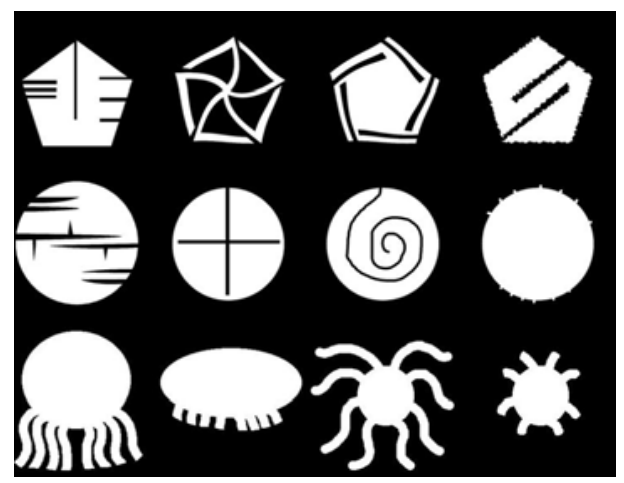

Fig. 3. Example shapes in MPEG-7 CE-Shape-1 part B database [1]

Table 1 presents the retrieval rates of our method and some classical descriptors. We see that the proposed method outperforms Shape Context when our method treats SC as the predefined descriptor. Besides, it outperforms some important works such as Inner-distance Shape Context (IDSC) 9] and Multi-scale Convexity Concavity (MCC) [6] as well. Our method also achieves a considerable retrieval rate compared with some recent works such as Triangle Area Representation (TAR) [11] and Shape Tree [10].

Table 1. Retrieval rates (Bullseye) of different methods on MPEG-7 CE-Shape-1 Part B data set [1]

\begin{tabular}{|c|c|}
\hline Algorithm & Score \\
\hline Shape Tree 10] & $87.70 \%$ \\
SC+Group-wised Matching+DP (Ours) & $\mathbf{8 7 . 1 5 \%}$ \\
TAR 11] & $87.13 \%$ \\
SC+DP 15] & $86.80 \%$ \\
IDSC+DP [9] & $85.40 \%$ \\
MCC 6] & $84.93 \%$ \\
Generative Models [7] & $80.03 \%$ \\
SC+TPS 3] & $76.51 \%$ \\
Visual Parts [2] & $76.45 \%$ \\
\hline
\end{tabular}

\subsection{Kimia's 99 Database}

The Kimia's 99 shape data set [5] is also very famous in shape matching and recognition. There are some occlusions, articulations and local deformations, thus it is suitable to be used to check both contour-based and skeleton-based shape descriptors. This data set includes ninety nine binary images from nine categories (see in Fig. 4). The retrieval result is summarized by counting the correct number of top 1 to top 10 nearest matches. The best possible result for each of them is 99 . 


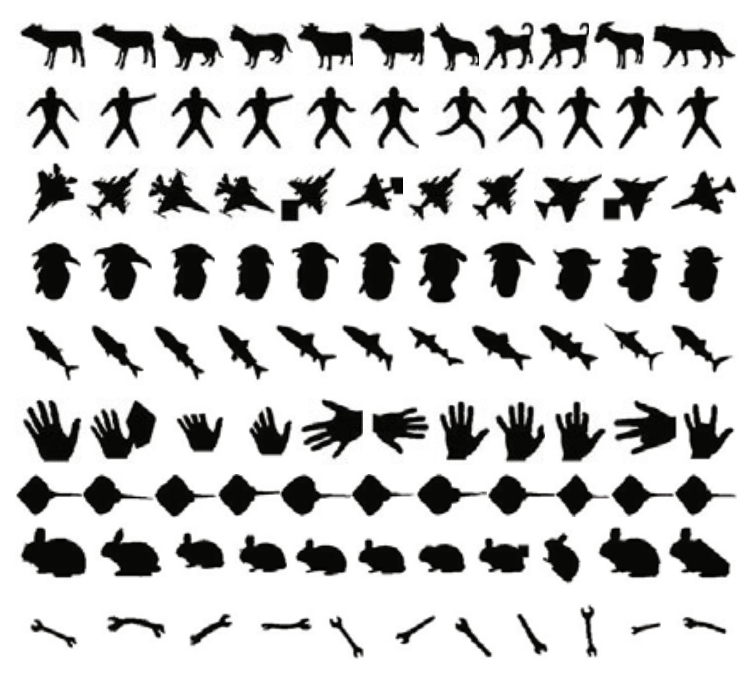

Fig. 4. The ninety-nine shapes in Kimia's 99 database [5]

Table 2. Retrieval results on Kimia's 99 data set [5]

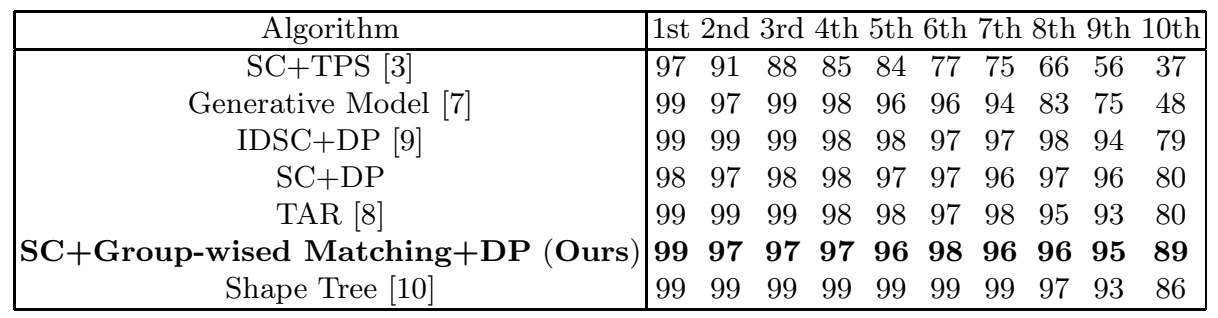

Table 2 gives the results of our method and some recent descriptors. Again, we find that our method outperforms Shape Context, and it is comparable with IDSC and TAR. The best result is achieved by Shape Tree [10].

\subsection{Tari1000 Database}

The Tari1000 data set 13 is also a large database for binary images. It consists of 1,000 binary images from 50 shape categories, i.e., 20 images per category, which is the same as in MPEG-7 database. Some of these categories are also included in MPEG-7 database, such as brick, cattle, cellular, phone,face, flatfish, fountain, key,ray, teddy, watch and so on. Figure 5 gives some examples for this data set. When testing the retrieval performance, we follow two rules, one is the "bulls-eye test" introduced in Section 3.1, and the other is the Precision-Recall (P-R) curve.

When following the "bulls-eye test", the score of our method (94.58\%) outperforms the result of Shape Context (94.18\% [15]). Figure 6 shows the PrecisionRecall curves of our method, Shape Context along with some other methods. 


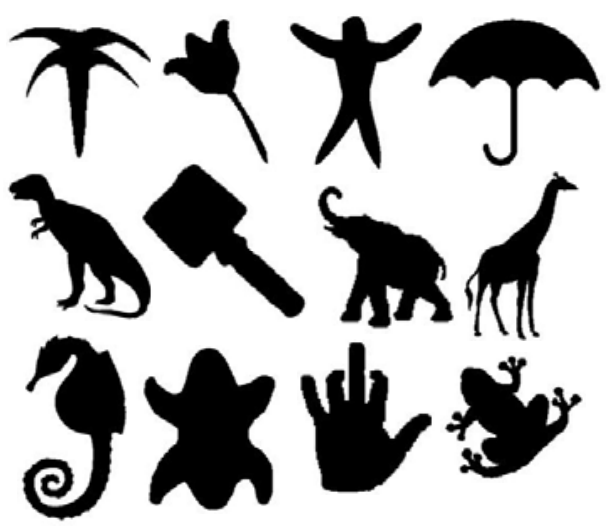

Fig. 5. Example shapes in Tari1000 database 13

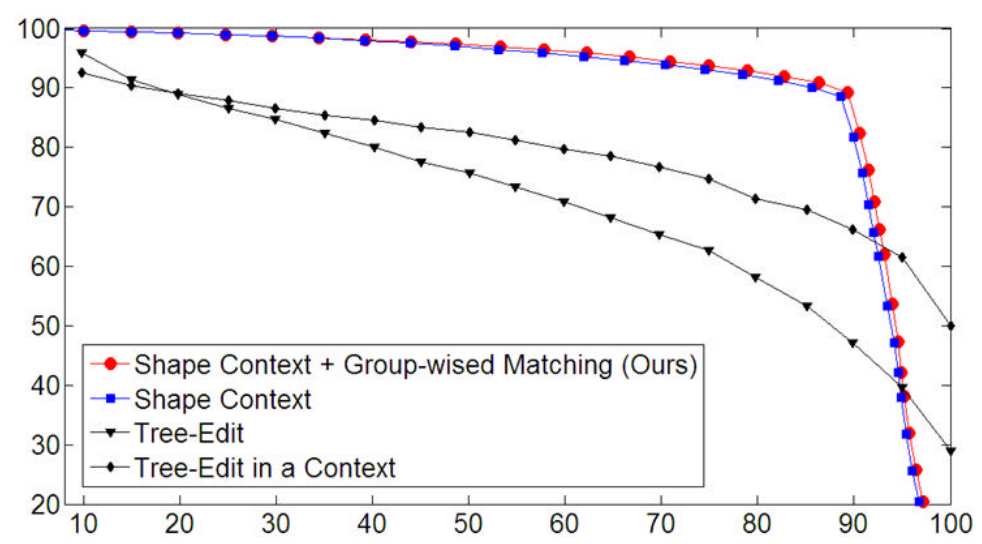

Fig. 6. Precision/Recall curves on Tari1000 database [13]

From the P-R curves we see that our method slightly outperforms Shape Context, and significantly outperforms the methods in 13 .

\section{Conclusions}

This paper presents one novel shape matching method called Group-wised Matching. As shown by its name, this method extends the usual pair-wised matching algorithm to a group-wised matching framework. One point is matched to another when the two corresponding groups achieves an excellent mapping. As the group of one contour sample point consists of its neighboring points, more shape characteristics along with the co-occurrence (order) information of the point sequence on the shape contour are included within the matching stage. As a result, the proposed matching method is able to obtain a more robust matching result. The robustness and power of our method has been demonstrated 
by the shape matching and retrieval experiments on three famous benchmarks: MPEG-7 data set, Kimia's data set and Tari1000 data set. The retrieval results show that the new group-wised matching method is able to get encouraging improvements compared to some traditional pair-wised matching approaches.

Several extensions of the proposed approach are possible. In this paper, the mapping cost for two groups is defined in a simple way, as every group is regraded as a short point sequence. In fact, the group may also be considered as a graph, and some current graph matching algorithms can be used to define the group-wised matching cost. Currently, the neighborhood or the size of the group (controlled by the parameter $k$ ) for every sample point are completely the same. This strategy, although very simple and easy to implement, may be improved. An algorithm may be invented to calculate one suitable value for the parameter $k$ for each sample point. Next, the group-wised manner may not only be used for contour points, but also be used for shape instances. Finally, our method may be extended to some other problems and applications such as point pattern matching [4] [12] and object detection in real images [16] 14].

Acknowledgments. The authors would like to thank Ling and Jacobs for releasing their source code of IDSC online. Parts of our work (such as boundary extraction, calculation of the Shape Context and Dynamic Programming algorithm) are accomplished by directly using corresponding parts of this code or slightly modifying them. The authors also would like to thank the anonymous referees who gave us many helpful comments and suggestions. This work was supported by National Natural Science Foundation of China \#60903096 and \#60873127.

\section{References}

1. Latecki, L.J., Lakamper, R., Eckhardt, U.: Shape Descriptors for Non-rigid Shapes with A Single Closed Contour. In: IEEE Conference on Computer Vision and Pattern Recognition (CVPR), pp. 424-429 (2000)

2. Latecki, L.J., Lakamper, R.: Shape Similarity Measure Based on Correspondence of Visual Parts. IEEE Transactions on Pattern Analysis and Machine Intelligence (TPAMI) 22, 1185-1190 (2000)

3. Belongie, S., Malik, J., Puzicha, J.: Shape Matching and Object Recognition Using Shape Context. IEEE Transactions on Pattern Analysis and Machine Intelligence (TPAMI) 24, 509-522 (2002)

4. Chui, H., Rangarajan, A.: A New Point Matching Algorithm for Non-rigid Registration. Computer Vision and Image Understanding (CVIU) 89(2), 114-141 (2003)

5. Sebastian, T.B., Klein, P.N., Kimia, B.B.: Recognition of Shapes by Editing Their Shock Graphs. IEEE Transactions on Pattern Analysis and Machine Intelligence (TPAMI) 25, 116-125 (2004)

6. Adamek, T., O'Connor, N.E.: A Multiscale Representation Method for Nonrigid Shapes with a Single Closed Contour. IEEE Transactions on Circuits and Systems for Video Technology (TCSVT) 14, 742-753 (2004)

7. Tu, Z., Yuille, A.L.: Shape Matching and Recognition - Using Generative Models and Informative Features. In: Pajdla, T., Matas, J(G.) (eds.) ECCV 2004, Part III. LNCS, vol. 3023, pp. 195-209. Springer, Heidelberg (2004) 
8. Alajlan, N., Rube, I.E., Kamel, M.S., Freeman, G.: Shape Retrieval Using Trianglearea Representation and Dynamic Space Warping. Pattern Recognition (PR) 40, 1911-1920 (2007)

9. Ling, H., Jacobs, D.W.: Shape Classification Using Inner-Distance. IEEE Transactions on Pattern Analysis and Machine Intelligence (TPAMI) 29, 286-299 (2007)

10. Felzenszwalb, P.F., Schwartz, J.D.: Hierarchical Matching of Deformable Shapes. In: IEEE Conference on Computer Vision and Pattern Recognition (CVPR), pp. 1-8 (2007)

11. Alajlan, N., Kamel, M., Freeman, G.: Geometry-based Image Retrieval in Binary Image Databases. IEEE Transactions on Pattern Analysis and Machine Intelligence (TPAMI) 30, 1003-1013 (2008)

12. Mcauley, J.J., Caetano, T.S., Barbosa, M.S.: Graph Rigidity, Cyclic Belief Propagation, and Point Pattern Matching. IEEE Transactions on Pattern Analysis and Machine Intelligence (TPAMI) 30(11), 2047-2054 (2008)

13. Baseski, E., Erdem, A., Tari, S.: Dissimilarity between Two Skeletal Trees in A Context. Pattern Recognition (PR) 42, 370-385 (2009)

14. Bai, X., Wang, X., Latecki, L.J., Liu, W., Tu, Z.: Active Skeleton for Non-rigid Object Detection. In: IEEE International Conference on Computer Vision (ICCV), pp. 575-582 (2009)

15. Bai, X., Wang, B., Wang, X., Liu, W., Tu, Z.: Co-transduction for shape retrieval. In: Daniilidis, K., Maragos, P., Paragios, N. (eds.) ECCV 2010, Part III. LNCS, vol. 6313, pp. 328-341. Springer, Heidelberg (2010)

16. Wang, B., Bai, X., Wang, X., Liu, W., Tu, Z.: Object Recognition Using Junctions. In: Daniilidis, K., Maragos, P., Paragios, N. (eds.) ECCV 2010, Part V. LNCS, vol. 6315, pp. 15-28. Springer, Heidelberg (2010) 